\title{
Optimization of automated electron diffraction tomography for challenging applications
}

\author{
$\underline{\text { Ute Kolb }}^{1}$, Yasar Krysiak ${ }^{1}$, Haishuang Zhao ${ }^{1}$, Sergi Plana-Ruiz ${ }^{1}$, Bastian Barton ${ }^{1}$ \\ ${ }^{1}$ Johhannes Gutenberg-University Mainz, Mainz, Germany \\ E-mail: kolb@uni-mainz.de
}

Ten years after the development of automated electron diffraction tomography (ADT) [1, 2] the analysis of the sequentially scanned reconstructed reciprocal space and the use of extracted reflection intensities for direkt crystal structure analysis has gained strong attention in many subjects. Especially in combination with precession electron diffraction allowing for reflection integration (ADT/PED) it was possible to extract fine structural details often explicitly important for physical properties of the material. The variety of nanocrystalline materials solved with ADT on the basis of quasi-kinematical electron diffraction intensity data covers alloys, large cell porous minerals, zeolites, beam-sensitive metal-organic frameworks and small organic molecules. Some originating from single nanocrystals down to $30 \mathrm{~nm}$, strongly agglomerated particles or FIB lamellae. In combination with other approaches like HR-TEM imaging, X-ray diffraction methods analysing reflections (XRPD) or total scattering information (PDF) and neutron diffraction (ND) or spectroscopic measurements like solid state nuclear magnetic resonance (SS-NMR) it was possible to describe the crystal structure solutions gained for twinned, pseudosymmetric or disordered material even more detailed [3].

ADT data can be in principle collected using standard TEMs but in order to collect electron diffraction data in a quality necessary to achieve high-end crystal structure solutions the data collection should be tailored to the material and the problem to be solved. Here we focus on the description of different approaches and the applicability to the various material classes.

[1] Kolb, U. \& T. Gorelik \& C. Kübel \& M.T. Otten \& D. Hubert, Ultramicroscopy (2007) 107, 507-513

[2] Kolb, U. \& T. Gorelik \& M.T. Otten, Ultramicroscopy (2008) 108, 763-772.

[3] Zhao H. \& Krysiak Y. \& Hoffmann K. \& Barton B. \& Molina-Luna L. \& Neder R.B. \& Kleebe H.-J. \& Gesing Th. M. \& Schneider H. \& Fischer R.X. \& Kolb U., J. Solid State Chem. (2017) 249, 114-123.

Keywords: electron diffraction, diffraction tomography, structure analysis 